\title{
Spatial and temporal air quality pattern recognition using environmetric techniques : a case study in Malaysia.
}

\begin{abstract}
The objective of this study is to identify spatial and temporal patterns in the air quality at three selected Malaysian air monitoring stations based on an eleven-year database (January 2000-December 2010). Four statistical methods, Discriminant Analysis (DA), Hierarchical Agglomerative Cluster Analysis (HACA), Principal Component Analysis (PCA) and Artificial Neural Networks (ANNs), were selected to analyze the datasets of five air quality parameters, namely: $\mathrm{SO}$, NO2, O3, $\mathrm{CO}$ and particulate matter with a diameter size of below $10 \mu \mathrm{m}$ (PM10). The three selected air monitoring stations share the characteristic of being located in highly urbanized areas and are surrounded by a number of industries. The DA results show that spatial characterizations allow successful discrimination between the three stations, while HACA shows the temporal pattern from the monthly and yearly factor analysis which correlates with severe haze episodes that have happened in this country at certain periods of time. The PCA results show that the major source of air pollution is mostly due to the combustion of fossil fuel in motor vehicles and industrial activities. The spatial pattern recognition (S-ANN) results show a better prediction performance in discriminating between the regions, with an excellent percentage of correct classification compared to DA. This study presents the necessity and usefulness of environmetric techniques for the interpretation of large datasets aiming to obtain better information about air quality patterns based on spatial and temporal characterizations at the selected air monitoring stations.
\end{abstract}

Keyword: Air quality; Multivariate analysis. 